\title{
Victimización de niños, niñas y adolescentes chilenos/as en la escuela
}

\section{Victimization among Chilean boys, girls and adolescents in schools}

\author{
Carlos Rodríguez-Garcés* (D) https://orcid.org/0000-0002-9346-0780 \\ Universidad del Bio-Bio, Chile, carlosro@ubiobio.cl \\ Geraldo Padilla (D) https://orcid.org/0000-0003-0882-1818 \\ Universidad del Bio-Bio, Chile, gpadilla@ubiobio.cl
}

Denisse Espinosa (1) https://orcid.org/0000-0003-1486-7046

Universidad del Bio-Bio, Chile, daespinosa@ubiobio.cl

*Autor para
correspondencia:
Carlos Rodríguez-Garcés
carlosro@ubiobio.cl

Recepción: 29/09/20

Aprobación: 26/03/21

Publicación: 13/07/21

\begin{abstract}
The aim of this article is to analyze the prevalence of the dimensions that characterize violence and victimization, typifying their manifestations on the basis of the observer/victim role, multidimensionality and context. Through an inferentialcomparative process with data from the First National Survey of Poly-victimization among Boys, Girls and Adolescents (2017), the transversal victimization and high-level exposure reported by BGA, especially regarding Common Crimes and Indirect Victimization, both at school and in the neighborhood, are exposed. Manifestations that make violence a complex, recurrent and ubiquitous phenomenon, whose approach demands the multidimensionality of agents and perspectives that involve family, neighborhood and school in the search for effective solutions.
\end{abstract}

Key words: poly-victimization, violence in schools, personal development, school community, childhood.

Resumen: El objetivo de este artículo es analizar las prevalencias de las dimensiones que caracterizan a la violencia y victimización, tipificando sus manifestaciones sobre la base del rol observador/ víctima, multidimensionalidad y contexto. Mediante un proceso inferencial-comparativo con datos de la Primera Encuesta Nacional de Polivictimización entre Niños, Niñas y Adolescentes (2017), se expone la transversalidad de la victimización y el alto nivel de exposición reportado por niños, niñas y adolescentes (NNA), especialmente respecto a delitos comunes y victimización indirecta, tanto en la escuela como en el barrio. Manifestaciones que hacen de la violencia un fenómeno complejo, recurrente y ubicuo, cuyo abordaje demanda la multidimensionalidad de agentes y perspectivas que involucren a la familia, el barrio y la escuela en la búsqueda de soluciones efectivas.

Palabras clave: polivictimización, violencia en la escuela, desarrollo personal, comunidad escolar, infancia. 


\section{Introducción}

Violencia, victimización e inseguridad se han transformado en temas comunes del discurso político y la preocupación ciudadana, teniendo como punto de encuentro la agenda de políticas públicas que cada gobierno y ministerios han propuesto durante las últimas dos décadas. La cobertura y análisis de estos fenómenos es motivo de interés para instituciones policiales y penales, organizaciones ciudadanas y centros de investigación, que advierten sobre el incremento en los niveles de comisión de algunos delitos y su atestiguamiento, reportándose niveles de violencia asociada que conmocionan a la opinión pública y derivan en la urgencia de prevenir la exposición (Tocornal et al., 2014; Fundación Paz Ciudadana, 2015).

El tratamiento que los medios de comunicación hacen de la delincuencia y victimización tampoco es alentador. La televisión, la radio y las redes sociales son activos productores de narrativas delictivas, y tras su aparente interés aséptico por develar y difundir las noticias, nutren el imaginario, ideas y prejuicios entre la ciudadanía respecto a la potencialidad de ser víctimas en cualquier lugar o momento.

$\mathrm{Al}$ igual que en otros países del mundo, en Chile se ha instalado con fuerza la cultura de la victimización o el pensar que, en algún momento y sin importar cómo o dónde, se está vulnerable (Pitch, 2009). Las víctimas y los victimarios recorren la parrilla televisiva e Internet, volviéndose parte de la vida social (Focás, 2013). En tal sentido, instituciones y medios de comunicación han concertado un interés paradójico, donde al tiempo que recogen esta inseguridad, la procesan e intentan reducir, también la extienden por múltiples sectores, volviéndola un fenómeno aparentemente ubicuo entre la ciudadanía.

\section{Violencia y victimización entre NNA}

Pese a lo que la mayoría piensa, la preocupación mediática y social por el bienestar físico y psicológico de NNA es un fenómeno relativamente reciente. Hasta hace poco, algunos análisis sobre violencia, delincuencia y victimización exponían que la raíz de la delincuencia, los disturbios y el vandalismo en las ciudades era la falta de control y contención de los jóvenes, especialmente pobres y de sectores marginales, quienes habían crecido entre la pobreza y el abandono, con un desprecio hacia el trabajo honrado y carentes de una figura paterna que los guiara (Beyer y Vergara, 2006). 
Solo un par de décadas atrás, y por muy extraño que parezca desde una visión moderna, los jóvenes arrastraban una percepción social y judicial de recelo, siendo actores constantes en los reportes de delitos agresivos, consumo de alcohol y drogas, uso de armas, deserción escolar y cultura de pandillas (Valdivieso, 1999). La forma en que se les evaluaba y trataba, en particular por las instancias de control social (policía, profesores, familia), estaba signada por un tutelarismo que se arrogaba la responsabilidad de su corrección. Aun a título de protegerlos, los jóvenes eran continuamente arrojados al entramado de vigilancia-intervención que constituían las estaciones de policía, los colegios, tribunales de justicia y reformatorios (Salmerón, 2011).

Sin embargo, y a pesar de lo lapidario de estos diagnósticos, a partir de mediados del siglo XX comenzó a tomar fuerza una perspectiva más "humana" en la comprensión y gestión de la población joven, sobre todo luego de la instalación de los Estados de bienestar y su búsqueda por mínimos vitales para la población (Bergalli y Rivera, 2007). Con el argumento de proteger su bienestar físico, psicológico y sexual, NNA pasaron progresivamente a considerarse víctimas de su entorno y condiciones de vida, con medidas institucionales que apelaban al apoyo de estos grupos a través de la asistencia y terapia.

Pese a este giro discursivo-práctico, se expandieron las redes de vigilancia, evaluación y pesquisa en torno a la infancia y adolescencia. Como bien exponen González (2014), para el caso de España, y Howell et al. (2015) respecto a Estados Unidos, el interés por los jóvenes pobres, vulnerables y potenciales víctimas se incrementó sustancialmente, lo mismo que los mecanismos y redes para captarlos, examinar su comportamiento y corregirlos.

En la actualidad, las comunidades escolares y barriales están cruzadas por distintas iniciativas para la identificación oportuna de violencia y victimización entre NNA. Los tutores, padres y madres han sido consultados e incluidos en la gestión de los colegios para extender los beneficios de un buen clima de convivencia escolar hacia el hogar y viceversa, ya que la primera socialización es crucial para la adquisición y ejercicio de pautas relacionales. Con esto, se han multiplicado los esfuerzos por vigilar y mantener bajo control a los jóvenes, regulando su comportamiento a través de acciones conjuntas entre instituciones de distinto nivel social (Esposito, 2010; Byung-Chul, 2014).

De pronto, la escuela, el barrio y la familia se convirtieron en lugares de constante evaluación y focos de atención para la política pública y social en materia juvenil. Algunas investigaciones y estudios develaban que la victimización y sus secuelas eran multifactoriales y multidimensionales (Valdenegro y Calderón-Flández, 2016), y no sólo atribuibles a la pobreza, el bajo capital 
social y la insuficiencia de redes. Así entendidas, la violencia y la vulnerabilidad eran transversales y polifacéticas, lo cual exigía actualizar su concepción y tratamiento legislativo hacia y entre NNA. Ya no se trataba solamente de robos, hurtos o ataques físicos, sino también de maltrato verbal en casa, insultos en la escuela, amenazas de daño en el barrio, exposición a balaceras y microtráfico de drogas en las calles, entre otros.

\section{La escuela como lugar de conflictos}

De todos los lugares, la escuela fue el sitio ideal para poner en marcha una serie de iniciativas que dejaran en claro la preocupación por el bienestar de NNA. Gracias al funcionamiento centralizado del sistema educativo, en 2009 y 2011 se iniciaron reformas para evaluar los climas de convivencia escolar, en particular sus dimensiones de seguridad, respeto y organización, por lo que el efecto dominó llegó hasta los rincones más alejados del territorio nacional (Agencia de Calidad de la Educación, 2017).

En poco tiempo, los colegios empezaron a publicar y compartir entre sus comunidades los protocolos de convivencia y las normas escolares antiviolencia, en el marco de un férreo compromiso con el desarrollo personal y social de los y las estudiantes. Situada en un contexto de creciente sensibilidad frente al delito y la victimización, la escuela trató de ser parte activa de las soluciones, funcionando como terminal de comunicación entre planes nacionales y adecuaciones locales para frenar lo que parecía una epidemia: la violencia (Tijmes, 2012).

Múltiples hallazgos de investigación advertían que la escuela estaba lejos de ser aquel sitio armónico donde el alumnado solamente debía preocuparse por aprender y compartir (Samper-García et al., 2015; Pérez-Archundia y Gutiérrez-Méndez, 2016). Ahí se suscitaban conflictos como peleas, robos, prácticas de aislamiento social, insultos y amenazas, que constituyen en su conjunto el núcleo duro de la violencia escolar, matonaje y bullying. En las investigaciones se encontró que en casos extremos todo esto puede llevar a que estudiantes -víctimas sistemáticas de estos actos- presenten secuelas en su autoestima, bajas habilidades sociales o ideaciones suicidas (Horna, 2017; Silva et al., 2017).

Respecto al contexto, hubo una especie de fijación hacia los sectores vulnerables que, pudiendo justificarse al principio por la atención focalizada que requerían, vino a transformarse prácticamente en el contexto de manifestación de la violencia en sí. Proliferaron los estudios comparativos que mostraban a la escuela pública y aquella que atendía a sectores de bajos 
recursos económicos y culturales como ejemplos paradigmáticos de la violencia desenfrenada, con agresiones a la orden del día y una naturalización de la violencia (Tijmes, 2012; Salgado, 2012; Castañeda, 2018). A esto se añadía el temor de directivos y profesores/as, quienes muchas veces se declaraban imposibilitados para actuar, lo cual contrastaba con la opinión de algunos estudiantes que veían a sus maestros/as como figuras autoritarias y estrictas (Tovar et al., 2016).

Entre todo esto, seguramente el argumento menos controversial era que la victimización, ya fuera en la escuela, el barrio o la casa, constituía un riesgo para el aprendizaje, el desarrollo personal y las trayectorias escolares. Por supuesto que esto también formó parte de la discusión, aunque a menudo fue más la base para decir que NNA requerían mayor intervención en lugar de plantear medidas íntegras para su atención. Si bien los planes multisectoriales aparentan altos grados de complejidad e interconexión entre diferentes actores, la fijación por mostrar productos más que entablar procesos de atención especializada opacó los esfuerzos. En otras palabras, con frecuencia fue más importante asegurar que existía un protocolo o plan de convivencia, que ofrecer a NNA un espacio seguro de convivencia cordial.

A esto debemos sumar las prácticas de segregación y segmentación escolar a lo largo y ancho del sistema educativo, lo cual justificaba pensar que aquellos colegios sin barreras de ingreso o requisitos para formar parte de la institución estaban a un paso de convertirse en lugares dominados por la anarquía de un grupo al que aún se veía con desconfianza. Con todo, así fue progresando la comprensión de la violencia y la victimización entre NNA; por una parte, se les veía como un grupo en oposición a las normas sociales, ad portas del caos y la comisión de delitos, y por otro, como personas vulnerables y sin voz, quienes tendrían la mala fortuna de vivir en condiciones de pobreza, habitar barrios problemáticos y haberse visto obligados a desertar tempranamente de la escuela.

Lo anterior ayuda a comprender el heterogéneo contexto donde aparecen y se aplican encuestas como la de Polivictimización en la Escuela (EPV). Entonces, ocupando los datos recogidos por la EPV entre NNA a nivel nacional respecto a victimización en la escuela, el barrio y la familia, el objetivo de este artículo es describir las distintas formas que adquiere la violencia y vulnerabilidad en este grupo; analizar los hechos que afectarían más el día a día de NNA, definiendo perfiles y distinguiéndolos por atributos sociodemográficos y algunos estructurales. Los resultados hacen especial énfasis en la escuela como contexto de victimizaciones, a la vez que destacan las diferentes posiciones de observador o victima directa que encarnan NNA. 


\section{Metodología}

\section{Diseño}

Este artículo fue elaborado desde una perspectiva metodológica cuantitativa. Se utilizan porcentajes, índices y medidas de tendencia central ( $x, D S)$ para describir el estado de victimización que reportan NNA entre $7^{\circ}$ básico y $3^{\circ}$ medio en Chile. Con fuentes secundarias de datos (BBDD), se analizan varias dimensiones e indicadores vinculados a la victimización, los cuales tienen impactos diferenciados en la población estudiantil según atributos como el género, la edad, ciclo educativo o tipo de colegio al que se asiste. Las BBDD corresponden a 2017 y se proyectan nuevas mediciones para 2021 y 2025 , por lo que el carácter transversal de nuestros resultados podrá ser robustecido con la disponibilidad de información más reciente.

\section{Descripción de la muestra}

La EPV se aplicó a 19.867 estudiantes de $7^{\circ}$ básico a $3^{\circ}$ medio pertenecientes a un total de 699 establecimientos. Con representación nacional, abarca las distintas tipologías de administración escolar e incluye las diversas regiones del país. El muestreo fue probabilístico estratificado, trietápico según establecimiento $>$ cursos $>$ estudiantes.

\section{Instrumento}

Los datos corresponden a la Primera Encuesta Nacional de Polivictimización en Niños, Niñas y Adolescentes (EPV-NNA) de 2017, encargada por la Subsecretaría de Prevención del Delito y el Consejo Nacional de Infancia a dos organismos técnicos pertenecientes a la Universidad de Chile: la Dirección de Estudios Sociales (DESUC) y el Programa de Estudios Sociales del Delito.

El cuestionario aplicado corresponde a una adaptación local del Juvenile Victimization Questionnaire (JVQ), desarrollado por Hamby et al., (2005), que se diseñó a fin de proveer a la investigación de un instrumento más comprensivo para la pesquisa de los distintos tipos de victimización que afectan a NNA. El JVQ ha sido aplicado mayoritariamente en Estados Unidos, aunque sus experiencias internacionales han demostrado consistencia y validez, teniendo adaptaciones en países como España (Pereda et al., 2014), Canadá (Cyr et al., 2013) y Reino Unido (Radford et al., 2013). 
Para el caso chileno se utilizaron 30 de los 34 indicadores originales y se añadieron dos ítems vinculados a la violencia en Internet, quedando finalmente organizados en seis dimensiones: Delitos comunes (7), Cuidadores (4), Pares (5), Sexuales (7), Indirectas (7) y Digitales (2). Se buscaba reportar tanto victimizaciones directas como indirectas por medio de un lenguaje accesible, especialmente respecto a eventos complejos como el abuso psicológico o sexual. El cuestionario fue autoaplicado con la asistencia de un facilitador y tiene un error muestral del \pm 0.7 .

\section{Procedimiento}

El análisis de los resultados de la EPV se divide en varios ejes. Primero, se exploran las variables que estructuran las diferentes dimensiones con que se mide la victimización entre NNA, analizándolas de forma desagregada según área: 1) Delitos comunes, 2) Cuidadores, 3) Pares, 4) Sexuales, 5) Indirectas y 6) Digitales.

Posteriormente, sobre la base de los 32 ítems que definen las tipologías de victimización, se establecieron dos índices aditivos según refieran los eventos al último año o a la vida. Con la información aportada por estos índices fueron construidos cuatro perfiles de polivíctima, utilizando la métrica de rango percentil y umbrales.

El primer método, recomendado por Hamby et al. (2005) para el análisis de la EPV, clasifica como polivíctima al percentil 90 o 92 de la distribución de victimizaciones, sea durante la vida o el último año, respectivamente. El segundo método se usa en experiencias más recientes, como la de Cyr et al. (2013) y Pereda et al. (2014), y se basa en el principio de acumulación de vivencias, estableciendo como punto de corte el umbral de cinco victimizaciones para ambos periodos.

Después se tipifican los diversos eventos de victimización de acuerdo con el rol de observador o víctima, segmentando las prevalencias detectadas sobre la base de atributos como edad, género y alguna dificultad física o intelectual. Con el objetivo de problematizar las experiencias de victimización en la escuela, se jerarquizan aquellas situaciones de mayor incidencia reportadas en este espacio, distinguiendo además entre contextos de educación municipal y con altos índices de vulnerabilidad (IVE).

Los resultados de la EPV fueron procesados según diseño de muestras complejas, determinando un ponderador para estudiantes [wgt_alu], control de estratos [var_strat] y conglomerados [var_unit]; dicho diseño busca reflejar la estructura original de la población a representar. 


\section{Resultados}

La exposición a eventos de victimización entre NNA es un hecho transversal y multidimensional. Así lo indican las tasas de prevalencia y promedios de cada una de las seis dimensiones que componen la EPV. En general, casi la totalidad de quienes participaron reportó haber vivido algún episodio de victimización durante su vida, es decir, 92,6\%; a pesar de que esta cifra descendió a 79,9\% para el caso del último año, resulta todavía muy preocupante. En promedio, NNA reportan 6,99 (0,059) victimizaciones durante la vida y $3,78(0,038)$ durante el último año.

Como se muestra en la Tabla $1,{ }^{1}$ cada dimensión de la EPV indica que NNA han estado expuestos a la vulnerabilidad en diferentes grados, siendo la victimización Indirecta (83,3\%), los Delitos comunes (77,0\%) y la Agresión de pares o hermanos/as (61,5\%) las situaciones de mayor prevalencia en su vida. Las medias estandarizadas por dimensión transitan de 0,226 a 0,409, haciendo referencia a riesgos de connotación sexual y a episodios de violencia indirecta, respectivamente.

En cuanto al último año, la preponderancia de las dimensiones mencionadas se reitera, aunque los índices de prevalencia disminuyen notoriamente en la razón de la franja temporal.

La distancia de las prevalencias observadas entre las dimensiones según el periodo de referencia que se consulte expresa la alta sensibilidad de la escala al momento de dimensionar la victimización. En atención a esto, se exponen índices estandarizados a fin de hacer comparables las dimensiones. De igual modo, se incluyen los ítems de mayor recurrencia durante el último año.

En lo referente a Delitos comunes -dimensión donde 51\% de NNA se declara víctima en alguno de los siete ítems-, encontramos que los eventos de mayor relevancia son los ataques físicos sin uso de armas $(28,5 \%)$ y el robo sin uso de fuerza (20,4\%), ambos con una recurrencia de al menos tres veces en el último año.

En cuanto a la victimización por cuidadores -donde uno de cada tres NNA $(34,8 \%)$ reporta vulneración en alguno de los cuatro ítems de la dimensión-, se observa también una alta incidencia, con un valor a nivel de escala que incluso supera al exhibido por la dimensión Delitos comunes $(0,354$ versus 0,278 , respectivamente). De los NNA que han sido vulnerados en el último año por situaciones de negligencia (82,2\%) o maltrato de cuidadores/as $(63,4 \%)$ lo ha sido en al menos tres ocasiones.

1 Todas las tablas y el gráfico se encuentran en el Anexo, al final del presente artículo (Nota del editor). 
Si bien las vulneraciones de índole sexual registran comparativamente una menor recurrencia, no dejan de ser preocupantes dada la naturaleza de los ítems que la conforman. Las prevalencias transitan entre 26,4\% y 16,7\%, según la franja temporal reportada. Un 7,9\% de NNA señala haber mantenido relaciones sexuales consentidas con alguna persona mayor de 18 años; de este porcentaje, $77,1 \%$ con una recurrencia de al menos tres veces en el último año.

Existe un conjunto de eventos que constituye un riesgo inminente para NNA, pese a no sufrir directamente esta situación de vulnerabilidad. La dimensión Victimización indirecta tiene el propósito de reflejar esta exposición, la cual, dada la convivencia deteriorada en algunos colegios y barrios, muestra alta prevalencia.

Una alta proporción de NNA indica haber sido testigo de una situación de maltrato o violencia durante su vida, y uno de cada tres $(32,2 \%)$ lo ha sido durante los últimos 12 meses. Las situaciones que más destacan son: testigos de balaceras, consumo y ventas de drogas, peleas de pandilla (45,3\%) y discriminación por raza, color de piel, discapacidad u orientación sexual (42,9\%); eventos que exhiben además altas y significativas recurrencias.

La dimensión de victimización Digital parte de la adaptación que se hizo del instrumento al contexto local y reporta bajos niveles de incidencia, con proporciones de exposición que transitan entre 32,3\% y 20,9\%, según el lapso temporal analizado. Un 14,1\% informó haber sido objeto de rumores o acoso por Internet, mientras que 11,8\% de NNA declaró haberse sentido incómodo/a por recibir preguntas de connotación sexual en alguna plataforma digital.

Este panorama general que entrega el análisis de las dimensiones puede ser profundizado con base en cada indicador que compone la EPV. Después de todo, la polivictimización como tal cobra sentido dependiendo del número de victimizaciones que reporte $10 \%$ u $8 \%$ superior de la distribución acumulada de hechos, ya sea durante la vida o el último año, respectivamente.

En el Gráfico 1 se muestra cómo la victimización decrece rápidamente al acumular ocurrencias. Entre quienes contestaron haber experimentado algún hecho de victimización durante su vida, 90,8\% lo ha sido entre una y 14 veces; para el caso del último año, 93,2\% de NNA manifestó haber vivido entre una y nueve victimizaciones.

Atendiendo a que la metodología fija la victimización sobre la base de un rango percentil poblacional, es decir, $10 \%$ superior en la distribución de victimizaciones durante la vida y $8 \%$ para los últimos 12 meses, en el caso chileno, NNA polivíctimas serían quienes durante la vida han experimentado 
al menos 15 eventos; mientras que en el último año esta tipología se configura a partir de los 11. Esto implica haber vivido cerca de la mitad (46,8\%) o un tercio $(34,3 \%)$ de los 32 hechos que componen la EPV durante la vida o el último año, respectivamente.

Dada la falta de sensibilidad del rango percentil para clasificar polivictimización, se ha utilizado de manera complementaria un criterio de segmentación por umbrales. Así, ya no se define la polivictimización sobre la base de la distribución, sino atendiendo al número de eventos. Se establece la polivictimización por umbrales a partir de la notificación de cinco eventos, generando subdivisiones de baja (entre cinco y seis indicadores) y alta (siete o más indicadores) polivictimización. En términos generales, las polivíctimas durante el último año representan 40,9\% de NNA, y 23,7\% se posiciona por sobre los siete indicadores.

Para profundizar en la caracterización de la polivictimización entre NNA, la Tabla 2 muestra cuáles son los indicadores más representativos, comparando las cifras generales con aquellos grupos considerados de riesgo: adolescentes, mujeres y quienes sufren alguna condición permanente y/o de larga duración.

De acuerdo con esta tabla, el rol preponderante entre NNA sería el de observadores de violencia, tipología que se acompaña con la de agresores y victimas en los análisis de conflicto escolar (Chamorro, 2018), y expresa la exposición o atestiguamiento de eventos peligrosos como manifestación del conflictivo contexto donde se vive o estudia. Un 45,3\% reporta haber presenciado episodios de balaceras, consumo de alcohol y drogas o peleas entre personas o pandillas, mientras que $42,9 \%$ ha sido testigo de discriminación. Cifras que tienden a ser significativamente más altas en los perfiles de comparación: mujeres, adolescentes y estudiantes con alguna condición parcial o permanente $(\mathrm{p}<0,01)$.

El segundo grupo de indicadores refiere al rol de víctimas e involucra situaciones que ponen en riesgo de manera directa la seguridad psicológica y física de NNA, observándose diferencias estadísticamente significativas entre los grupos de comparación $(\mathrm{p}<0,01)$. Entre estos siete indicadores destacan el ataque físico sin uso de armas $(28,5 \%)$, el robo sin uso de fuerza $(20,4 \%)$ y los golpes o ataques físicos por otros NNA (14,8\%). Dichos indicadores concitan especial preocupación, debido a que con relativa independencia de la subjetividad o creencia de las víctimas son eventos que ponen en riesgo la estabilidad de la comunidad educativa, la efectividad de sus mecanismos dialógicos para la resolución de conflictos y la imagen de seguridad ante el público. 
Respecto a los perfiles de comparación, entre los adolescentes mayores de 15 años lo que más se repite es la percepción de abandono o agresividad verbal de algún adulto cercano (29,3\%); entre mujeres fue el ataque físico $(31,8 \%)$; y para quienes declararon alguna condición permanente y/o de larga duración, ambas victimizaciones fueron manifestadas en similar proporción (37,3\% y $35,3 \%$, respectivamente). En cuanto a los tres indicadores que involucran de forma directa a los pares como agresores, sea por golpes, amenazas de daño físico o insultos/exclusión, encontramos que el grupo más vulnerable es el de quienes manifiestan alguna condición permanente y/o de larga duración ( $\geq 20,0 \%)$.

Con base en estos resultados, y en razón del contexto educativo donde se lleva a cabo la EPV, emerge claramente la pregunta sobre cuáles son las características de la victimización referida al colegio. La Tabla 3 expone los eventos de vulneración más recurrentes en el espacio escolar, distinguiendo en razón de atributos estructurales. A nivel general, nueve de los 32 indicadores que componen la EPV registran prevalencias por sobre $50 \%$ al interior del espacio escolar, caracterizando los climas de convivencia entre los establecimientos participantes.

En el contexto escolar chileno se evidencian episodios de violencia tanto verbal como física, transitando por experiencias de exclusión social, coacción y agresiones de variada índole. Concretamente, durante el último año, los episodios de victimización más comunes fueron el malestar por insultos o exclusión de otros NNA (91,9\%), la imposición a hacer cosas que no se quiere $(79,4 \%)$ y recibir golpes, ataques o amenazas por alguna característica personal $(78,0 \%)$.

$\mathrm{Al}$ consultarles respecto a la frecuencia con que enfrentaban situaciones de victimización, NNA reportaron haber vivido u observado en más de una ocasión al mes discriminación (51,0\%), golpes, ataques o amenazas por caracteristicas personales $(38,5 \%)$ y malestar por insultos o exclusión (35,4\%). En cuanto a los atributos estructurales de las unidades educativas, no encontramos diferencias significativas. La victimización se evidencia como un fenómeno presente en múltiples comunidades educativas, exhibiendo prevalencias homogéneas entre los distintos tipos de administración escolar y niveles de vulnerabilidad estudiantil con que deben trabajar las instituciones.

El análisis de la EPV expone la multidimensionalidad de la vulnerabilidad que enfrentan algunos/as NNA en su espacio escolar, comunitario y familiar. Es preocupante la predominancia de eventos relacionados a daño físico directo, como golpes, ataques o robo, los cuales, pese a no involucrar el uso de armas u objetos similares, constituyen un perjuicio para el bienestar personal y colectivo de las comunidades educativas. 


\section{Discusión}

La aplicación de instrumentos como la EPV responde al creciente interés público en torno a las experiencias de victimización y percepción de inseguridad entre la ciudadanía. Por supuesto, el sistema educativo no se encuentra exento de esfuerzos para prevenir y sancionar la violencia en sus diferentes manifestaciones, especialmente por la responsabilidad social y formativa que tiene la escuela.

En términos generales, observamos que muy pocos NNA declaran no haber estado expuestos a algún tipo de agresión física o verbal en su ambiente escolar, barrial o familiar durante su vida o el último año. Sin embargo, no estuvieron ajenos al atestiguamiento de hechos de discriminación, peleas o robos en los lugares que habitan cotidianamente. Este primer acercamiento, amplio y referido a las dimensiones que componen la EPV, da cuenta de un riesgo o vulnerabilidad ubicua, donde a la discusión por el estado de victimización entre NNA se añade la preocupación por conocer cuáles son los eventos que suponen mayor peligro para estos grupos.

En tal sentido, del extenso grupo de indicadores que componen la encuesta, destacan aquellos vinculados a la violencia directa y el daño físico, como los ataques por otro grupo de NNA o los robos. Aun cuando estos eventos puedan prescindir del uso de objetos o armas, estamos hablando de un tipo de violencia que involucra contacto, forcejeo, resistencia y disputas, todo lo cual afecta el desarrollo personal y social de los y las jóvenes. Si bien las huellas corporales o físicas de este tipo de agresiones pueden no perdurar en el tiempo, sí lo hacen las huellas psicológicas del evento, el sentimiento de vulnerabilidad, las cuales impactan negativamente en la autoimagen, autoconcepto y sentimiento de seguridad (Massenzana, 2017).

Cabe destacar que nuestros resultados son consistentes con lo expuesto por otras investigaciones que utilizaron y/o adaptaron la EPV en latitudes tan distantes como España (Pereda et al., 2014), China (Chan et al., 2013) o Pakistán (Bashir y Dasti, 2015). Al igual que en el contexto local, estos trabajos ponen de manifiesto dos características generales de cómo se comporta la victimización entre NNA. Primero, que la diversificación de dimensiones es útil para comprender la victimización como una condición de vida en lugar de un evento aislado, en especial para quienes forman parte de una población vulnerable (sea por edad, capital económico o redes). Lo segundo es que debido a la posición y percepción que se tiene de la población joven no existe una línea que separe las agresiones físicas, psicológicas, verbales o sexuales, es 
decir, NNA son receptores de una violencia multidimensional que mezcla y cruza distintas dimensiones (Pinto-Cortez et al., 2017). En síntesis, la victimización puede llegar a formar parte de la cotidianidad en algunos grupos y estar afectando simultáneamente su desarrollo físico, psicológico y/o sexual.

En cuanto a las dimensiones como tales que forman parte de la EPV, para el caso chileno se repiten los Delitos comunes, en particular durante el último año, y el atestiguamiento de eventos de riesgo, prevalentes al preguntar por la vida. También es relevante la vulnerabilidad percibida o vivida por Cuidadores y por Pares durante la vida o los últimos 12 meses, pues si bien las cifras no son tan abultadas, reportan una exposición al riesgo en dos de los grupos más íntimos de relación social y afectiva: la familia y los amigos/ cercanos. Dado que la infancia y adolescencia se caracterizan por la necesidad de apoyo y herramientas que los cuidadores puedan proveer, además de los modelos y comportamientos que se ejercen entre los grupos de referencia, estos cuatro tipos de victimización dificultarían la tarea de mitigar, controlar y corregir eventos de violencia o daño hacia la población joven.

Tal vez una de las consecuencias más frecuentes de la exposición prolongada a la violencia entre población joven sea la naturalización o desensibilización frente a la recepción, ejercicio o atestiguamiento de la misma. Hay varios argumentos para esta afirmación, entre los que destacan, incluso, la apreciación futura de la violencia como medio legítimo para la defensa personal, colectiva o barrial, especialmente en contextos donde existe crimen organizado o cultura de pandillas (Castaño y Loaiza, 2018). El aspecto simbólico de la violencia se despliega con mayor facilidad entre los grupos desensibilizados, los cuales no sólo están habituados a vivirla, sino que llegan a justificar su manifestación como parte del lugar donde viven y del modo en que comprenden cómo se lleva a cabo la vida (Galán, 2018). Por último, en algunos casos, esta naturalización de la violencia puede acarrear la percepción de la misma como medio para ascender en la escala de liderazgo entre grupos, con lo cual se reduce progresivamente la eficacia de medidas tendientes a la pacificación y/o protección de los y las jóvenes.

Ahora bien, para ser justos, existe una relación problemática entre la visión de NNA como víctimas de delitos comunes, mostrada por instrumentos oficiales como la EPV, y aquella vinculada al tratamiento sociomediático de este grupo en relación con la comisión de delitos. En Chile, tal y como sucede en múltiples países, no sólo hay inconvenientes para definir qué es ser víctima -de allí la sensibilidad que tiene el instrumento para tipificar esta condición-, sino también para saber cuál es la valoración adecuada hacia los y las jóvenes. La victimización por delitos y el atestiguamiento de violencia 
entre estos grupos contrasta notoriamente con la culpabilización que reciben desde amplios sectores.

En periódicos, programas de radio y reportajes de televisión suele involucrarse a población joven con delitos, drogas y resistencia a las normas comunes de convivencia pacífica. De un tiempo hacia acá se ha vuelto usual ver en los medios de comunicación los relatos de víctimas y expertos advirtiendo a la comunidad sobre la precocidad de quienes roban o agreden, tanto en los espacios públicos como residenciales. Se ha apuntado sistemáticamente a los jóvenes como delincuentes, aludiendo a su decadencia de valores y disciplina, o que al buscar alternativas fáciles para escapar de la pobreza hacen de la delincuencia, las drogas y las armas un estilo de vida (Loeber et al., 2011; Castaño y Loaiza, 2018).

Más allá de los argumentos que se puedan esgrimir para apoyar o desmentir este tipo de discusiones, hay que agregar un poco de perspectiva socioterritorial en todo esto, a fin de ampliar los criterios para la formación de un juicio. Pese a la ubicuidad de la delincuencia y la violencia reportadas a nivel nacional por la EPV, su frecuencia e intensidad se correlacionan - a juicio de varias investigaciones- con la disponibilidad de recursos económicos y sociales de las familias que habitan un territorio, barrio o sector de las ciudades (Burgess, 2009; Trucco y Inostroza, 2017). En la percepción pública y en la de las instituciones y los programas de prevención del delito hay una relación tácita entre periferia y mayor exposición a victimizaciones por robo, hurto o peleas callejeras. Aunque con exageraciones por parte de los medios de comunicación, estos lugares tienden a convertirse en guetos definidos por el crimen, la segregación y la pobreza (Ortega, 2014), todo lo cual dificulta romper los ciclos de violencia y vulneración entre la población joven.

Así entendido, la relación entre los indicadores de la EPV y las posibles soluciones se complica cuando agregamos las características de estos contextos territoriales. Mientras algunos NNA pueden estar habitando el limbo de víctimas-victimarios, cifras oficiales los consideran envueltos por el riesgo, y al mismo tiempo se les apunta, simbólica o mediáticamente, como perpetradores de actos similares. Según la EPV, los delitos comunes son la mayor amenaza para NNA: robos, ataques y amenazas; en tanto, para la institución policial, los jóvenes son los principales responsables de los delitos clasificados como de mayor connotación social, es decir, robos, asaltos o hurtos (Kessler y Dimarco, 2013; Fundación Paz Ciudadana, 2015). En otras palabras, NNA serían víctimas y victimarios de robos, hurtos y ataques, lo cual, en lugar de ser algo favorable para la adecuada comprensión y abordaje de la problemática, se convierte regularmente en evidencia para su estigmatización y vinculación con el delito (Rodríguez et al., 2017). 
Volviendo al resto de dimensiones de la EPV, encontramos que los indicadores de victimización sexual alcanzan una baja magnitud comparada; sin embargo, constituyen una situación preocupante dado el carácter delictivo de estos eventos. Como vimos, alrededor de dos de cada 10 NNA (16,7\%) declararon haber estado expuestos a prácticas u hostigamiento sexual durante los últimos 12 meses, y una proporción significativa de ellos/as declaró haber vivenciado esto más de una vez al mes. A diferencia de otros estudios internacionales (Pereda y Forns, 2007; Chan et al., 2013), donde la pregunta respecto a prácticas sexuales consentidas con mayores de 18 años fue omitida o sustituida por la pesquisa de abuso sexual infantil, en el caso chileno se muestra que es un evento de escasa ocurrencia, pero de alta intensidad entre quienes lo reportan.

La atención social y política que han tenido los hechos de violencia sexual durante la última década, en especial los ejercidos contra mujeres y niños/as, han llevado a incrementar los mecanismos y recursos dirigidos a su prevención, reducción y sanción. Debido a los escándalos ampliamente difundidos por los medios de comunicación, y que dejaron al descubierto la vulneración o abuso de NNA en nuestro país -entre los que destacan los abusos sexuales vinculados a la Iglesia católica o a instituciones de protección infantil-, la preocupación social y judicial por los derechos sexuales entre NNA se incrementó.

Para continuar con uno de los objetivos principales de la EPV -el perfilamiento por rango percentil de la polivictimización entre NNA-, encontramos que para el caso chileno ésta se tipifica a partir de 14 indicadores durante la vida y 10 para el último año, lo cual es similar a lo exhibido en países como Reino Unido (Radford et al., 2013) y Estados Unidos (Finkelhor et al., 2011). Respecto a la polivictimización por umbrales, detectamos diferencias elevadas con otros estudios. Por ejemplo, utilizando el criterio de cinco o más indicadores como base del perfil polivíctima, Chile reporta 40,9\% de NNA bajo esta condición, mientras que estudios en Finlandia y Canadá exhiben cifras que no superan 10\% (Ellonen y Salmi, 2011; Cyr et al., 2013).

Esto pone de manifiesto los rasgos culturales de la victimización entre NNA, un fenómeno de preocupación internacional, pero con resguardos locales que exige adaptabilidad a las medidas de acción. A las necesidades universales de la población infantil y adolescente, como protección, cuidado, afecto y apoyo, se deben incluir aquellas que le dan sentido a la vida particular de los grupos, como puede ser la imbricación con rasgos culturales autóctonos, la implicación en actividades comunitarias o barriales o la valoración por 
prácticas religiosas. Si bien las polivíctimas representan el lado más crudo de la violencia como una condición de vida, no es menos importante recordar que el daño o perjuicio a la población infantil y adolescente deja huellas en su potencial humano, académico o intelectual, especialmente entre quienes ya ven limitados sus recursos debido al contexto de desigualdad y pobreza donde crecen (de Castro et al., 2013).

En tal sentido, el caso chileno preocupa no sólo porque la vulneración múltiple o polivictimización durante la infancia y/o adolescencia pone en riesgo el bienestar y desarrollo presente y futuro de NNA (Chen et al., 2010; Acosta et al., 2017), sino por las características de morbilidad asociada que tiene la violencia. Quienes son testigos de balaceras, robos o peleas también pueden estar siendo víctimas de estos delitos; más aún, quienes son víctimas de ataques físicos y violencia por parte de cuidadores o pares muy probablemente lo son también de insultos, descalificaciones, burlas y aislamiento. El análisis multidimensional de la victimización deja entrever estas problemáticas, vislumbrando de paso que tales hechos no pertenecen tan sólo a la vía pública como el imaginario social apela, sino también a la escuela y el hogar (Finkelhor et al., 2007).

En términos específicos, las tres primeras situaciones de victimización más reportadas corresponden a la exposición o atestiguamiento de balaceras, discriminación y ataques físicos sin armas (Tabla 2). Tal y como otros hallazgos de investigación indican (Contreras et al., 2016; León et al., 2019), es más sencillo reportar la violencia observada que la vivida, sobre todo en la escuela y el hogar, al ser grupos vulnerables: los adolescentes, las mujeres y quienes presentan alguna necesidad educativa especial. Con esto podemos afirmar que el rol preponderante entre NNA sería el de observadores de violencia, tipología que se acompaña con la de agresores y victimas en los análisis de conflicto escolar (Chamorro, 2018).

En el segundo grupo de indicadores más recurrentes durante el último año, donde NNA encarnan el rol de víctimas directas, se reiteran los hechos que ponen en riesgo la seguridad personal y física. Mientras el primer conjunto de indicadores permite alternativas de actuación, como buscar ayuda, huir o intervenir (Cano y Vargas, 2018; Pacheco, 2018), en el segundo se dificulta la reacción pacífica. Estas situaciones concitan especial preocupación debido a que, con relativa independencia de la subjetividad o creencia de algunas víctimas que desestiman las agresiones, implican un riesgo para la comunidad educativa entera. Además de alimentar el ciclo de agresiones, la pasividad frente a estos eventos denota incapacidad en las instituciones y equipos profesionales para instalar y gestionar adecuados climas de convivencia, dando 
cabida a que prácticas como bullying y acoso escolar perduren (García, 2010; Barcaccia et al., 2017).

La tercera y última parte de los resultados estuvo destinada al análisis del contexto donde ocurre la victimización. Así como no es lo mismo ser víctima de un ataque o robo que presenciar insultos o garabatos, no es lo mismo sufrir de victimización en el barrio o el hogar que en la escuela. En esta última existen, o deberían estar disponibles, profesionales dedicados al apoyo educativo y social de NNA, ya que la escuela es un espacio de protección y desarrollo integral. No obstante, debido a la naturaleza de la violencia reportada y a la prolongada permanencia en el espacio escolar, la escuela es el escenario más probable para conflictos y victimización entre NNA.

De este modo, eventos recurrentes como el robo sin uso de fuerza, el destrozo de objetos personales y golpes o amenazas por parte de otros NNA tienden a situarse en la escuela, e inclusive a no indicar, salvo excepciones, diferencias significativas por atributos estructurales como la dependencia y el nivel de vulnerabilidad socioeconómica del establecimiento. Si bien algunas investigaciones tienden a localizar los hechos o percepción de violencia particularmente en colegios públicos y sectores vulnerables (Guerra et al., 2012; Tijmes, 2012; González y Bassaletti, 2016), la ausencia de diferencias estadísticamente significativas en nuestro caso se explicaría por la alta sensibilidad y escasa capacidad de discriminación que tiene la EPV.

Por último, los datos refuerzan la premisa de organizar medidas multisectoriales y multidimensionales, a fin de reducir los hechos de violencia y victimización que pueden vivir o presenciar NNA. No se trata sólo de evitar que sufran daños corporales, sino, en especial, de evitar perjuicios psicológicos durante etapas que resultan cruciales para el desarrollo de futuros ciudadanos y ciudadanas (Hamby et al., 2005). La seguridad en la escuela es sólo un cariz al momento de plantear medidas, ya que, como vimos, la relación con los cuidadores y pares que se despliega en el hogar o el barrio también afectan el bienestar infantil y adolescente. Uno de los principales retos en esta temática es lograr que la victimización e inseguridad no se vuelvan experiencias constitutivas de la cotidianidad y de la forma en que nos relacionamos como miembros de una sociedad, sino como una problemática que requiere ser diagnosticada y enfrentada con los instrumentos y programas adecuados. 


\section{Conclusiones}

La victimización entre NNA es un evento de interés social cuyas implicancias traspasan el ámbito personal y se instalan en la agenda de políticas públicas. Sus efectos no sólo repercuten sobre el desarrollo presente y futuro de las víctimas y victimarios, sino que también amenazan la convivencia democrática y la resolución de conflictos. Al ser la escuela, el barrio y la familia espacios primordiales de socialización, la identificación oportuna de eventos de victimización facilita la acción temprana, protegiendo tanto a NNA como a la comunidad que los alberga.

Si bien las experiencias de victimización reportadas por la EPV son variadas en tipología, magnitud y prevalencia entre NNA, algunas de sus manifestaciones son tanto o más importantes que su recurrencia observada. Así, por ejemplo, aun cuando las agresiones sexuales reportan baja incidencia comparada entre NNA, constituyen una situación traumática de alto impacto que genera inseguridad y desconfianza. De igual modo, las agresiones de cuidadores resultan preocupantes, ya que las víctimas pueden llegar a naturalizar la violencia y su ejercicio como mecanismo de resolución de conflictos. Por último, la ubicuidad de los reportes de victimización plantea la cuestión de la violencia como un rasgo presente en múltiples relaciones, sea en la escuela, el barrio o la familia, lo cual conlleva el riesgo de modelar la violencia o victimización como inherente a la cultura.

Los hallazgos de este trabajo apuntan a la implementación de medidas multisectoriales que aúnen recursos y actores en torno al tratamiento de la victimización como fenómeno complejo. El crecimiento personal y social, elemento clave de los nuevos marcos de medición de calidad educativa, requiere asegurar espacios libres de agresión y violencia, reforzar políticas educativas para su prevención, manejo y derivación, potenciando las relaciones entre la escuela, la familia y el barrio.

\section{Limitaciones}

Una de las principales limitaciones de este estudio es la forma en que se estima la victimización entre NNA en la EPV. Si bien los 32 indicadores que la componen registran una amplia cobertura temática, su expresión binomial de presencia/ausencia exhibe una escasa capacidad discriminativa. La escala asigna igual valoración entre ítems con independencia de su naturaleza, y no consigna la recurrencia de su manifestación en la estimación métrica. De este modo, el atestiguamiento tiene el mismo valor que ser víctima directa; los 
Carlos Rodríguez-Garcés, Geraldo Padilla y Denisse Espinosa Victimización de niños, niñas y adolescentes chilenos/as en la escuela

insultos computan idéntico a la agresión sexual; asimismo, no se establecen diferencias entre el evento acontecido una vez al año respecto de aquellos más frecuentes.

Atendiendo a la riqueza de la información contenida en la EPV y su validez poblacional, sería de interés mejorar los índices de clasificación y sensibilidad de la escala por medio de una graduación de los ítems sobre la base de su naturaleza, intensidad y recurrencia.

\section{Referencias}

Acosta, Esther et al. (2017), "Conocimientos sobre maltrato infantil en adolescentes maltratados y padres o tutores", en Revista Cubana de Pediatría, vol. 89, núm. 2, Cuba: Ciencias Médicas.

Agencia de Calidad de la Educación (2017), Resultados educativos 2017, Chile: Agencia de Calidad de la Educación.

Barcaccia, Bárbara et al. (2017), "Bullying and the detrimental role of un-forgiveness in adolescents wellbeing”, en Psicothema, vol. 29, núm. 2, España: Colegio Oficial de Psicólogos del Principado de Asturias.

Bashir, Zoahib y Dasti, Rabia (2015), "Poly-victimization and mental health of street children in Lahore city”, en Journal of Mental Health, vol. 24, núm. 5, Estados Unidos: Taylor \& Francis.

Bergalli, Roberto y Rivera, Iñaki (2007), Jóvenes y adultos. El difícil vínculo social, España: Anthropos.

Beyer, Harald y Vergara, Rodrigo (2006), Delincuencia en Chile: determinantes y rol de las politicas públicas, Chile: Instituto de Economía, Pontificia Universidad Católica de Chile.

Burgess, Rod (2009), “Ciudad, espacio público y convivencia”, en Falú, Ana [ed.], Mujeres en la ciudad: de violencia y derechos, Chile: Ediciones SUR.

Byung-Chul, Han (2014), La agonía del Eros, España: Herder.

Cano, Margarita y Vargas, Jorge (2018), “Actores del acoso escolar”, en Revista Médica de Risaralda, vol. 24, núm. 1, Colombia: Universidad Tecnológica de Pereira.

Castañeda, Giovanny (2018), “Maltrato escolar, práctica pedagógica y lenguaje”, en Enunciación, vol. 23, núm. 2, Colombia; Universidad Distral Francisco José de Caldas.

Castaño, Stiven y Loaiza, Miguel (2018), "Naturalización de la violencia urbana: representaciones sociales en estudiantes de Medellín, Colombia”, en Voces y Silencios: Revista Latinoamericana de Educación, vol. 9, núm. 2, Colombia: Universidad de los Andes.

Chamorro, Sara (2018), Violencia escolar en estudiantes de Educación Secundaria de Valparaiso (Chile): comparación con una muestra de Zaragoza (España), España: Facultad de Ciencias Sociales y del Trabajo.

Chan, Ko et al. (2013), "Associating child sexual abuse with child victimization in China", en The Journal of Pediatrics, vol. 162, núm. 5, Estados Unidos: Association of Medical School Pediatric Department Chairs. 
Convergencia Revista de Ciencias Sociales, vol. 28, 2021, Universidad Autónoma del Estado de México

Chen, Laura et al. (2010), "Sexual abuse and lifetime diagnosis of psychiatric disorders: systematic review and meta-analysis”, en Mayo Clinic Proceeding, vol. 85, núm. 7, Estados Unidos: Clinic Mayo.

Contreras, Dante et al. (2016), "Bullying, identity and school performance: Evidence from Chile”, en International Journal of Educational Development, vol. 51, núm. 1, Estados Unidos: Elsevier.

Cyr, Katie et al. (2013), "Polyvictimization and victimization of children and youth: Results from a populational survey”, en Child Abuse \& Neglect, vol. 37, núm. 10, Estados Unidos: Elsevier.

de Castro et al., Filipa (2013), "Indicadores de bienestar y desarrollo infantil en México", en Salud Pública de México, vol. 55, núm. 2, México: Instituto Nacional de Salud Pública.

Ellonen, Noora y Salmi, Venla (2011), "Poly-Victimization as a Life Condition: Correlates of Poly-Victimization among Finnish Children”, en Journal of Scandinavian Studies in Criminology and Crime Prevention, vol. 12, núm. 1, Suecia: Universidad de Estocolmo.

Esposito, Roberto (2010), Communitas: the origin and destiny of community cultural memory in the present, Estados Unidos: Stanford University Press.

Finkelhor, David et al. (2007), "Poly-victimization: A neglected component in child victimization”, en Child Abuse \& Neglect, vol. 31, núm. 1, Estados Unidos: Elsevier.

Finkelhor, David et al. (2011), "Polyvictimization in developmental context", en Journal of Child \& Adolescent Trauma, vol. 4, núm. 4, Suiza: Springer International Publishing.

Focás, Brenda (2013), “Inseguridad: en busca del rol de los medios de comunicación”, en La trama de la comunicación, vol. 17, Argentina: Universidad Nacional de Rosario.

Fundación Paz Ciudadana (2015), Balance de la delincuencia en Chile 2014: Radiografía del sistema de seguridad y justicia, Chile: Fundación Paz Ciudadana.

Galán, Jaime (2018), "Exposición a la violencia en adolescentes: desensibilización, legitimación y naturalización”, en Diversitas, vol. 14, núm. 1, Colombia: Universidad Santo Tomás.

García, José (2010), “Bullying”, en Innovación y Experiencias Educativas, núm. 27, España: Central Sindical Independiente y de Funcionarios.

González, Sonia (2014), Jóvenes delincuentes: un análisis criminológico de tres historias de vida. Treballs Finals de Grau de Criminologia, España: Universitat de Barcelona.

González, Patricia y Bassaletti, Rodrigo (2016), "Convivencia y prevención de violencia escolar: los desafíos que vienen para Chile”, en Conceptos, núm. 35, Argentina: Universidad del Museo Social Argentino.

Guerra, Cristóbal et al. (2012), "Percepción del clima escolar en estudiantes de enseñanza media de Valparaíso de colegios municipales, particulares subvencionados y particulares”, en Estudios Pedagógicos (Valdivia), vol. 38, núm. 2, Chile: Universidad Austral de Chile.

Hamby, Sherry et al. (2005), The Juvenile Victimization Questionnaire (JVQ): Administration and scoring manual, Estados Unidos: Crimes Against Children Research Center.

Horna, Víctor (2017), "Un estudio cualitativo sobre convivencia escolar: el bullying desde la perspectiva de las víctimas", en ConCiencia EPG, vol. 2, núm. 2, Perú: Universidad Nacional de Educación Enrique Guzmán y Valle.

Howell, James et al. (2015), "Serie especial: transición desde la delincuencia juvenil a la delincuencia adulta”, en Revista Española de Investigación Criminológica, vol. 2, núm. 13, España: Sociedad Española de Investigación Criminológica. 
Kessler, Gabriel y Dimarco, Sabina (2013), “Jóvenes, policía y estigmatización territorial en la periferia de Buenos Aires”, en Espacio Abierto, vol. 22, núm. 2, Venezuela: Universidad del Zulia.

León, Celeste et al. (2019), "Victimización y violencia escolar: el rol de la motivación de venganza, evitación y benevolencia en adolescentes”, en Revista de Psicodidáctica, vol. 24, núm. 2, España: Elsevier.

Loeber, Rolf et al. (2011), "La transición desde la delincuencia juvenil a la delincuencia adulta”, en Revista Española de Investigación Criminológica, vol. 9, España: Sociedad Española de Investigación Criminológica.

Massenzana, Florencia (2017), "Autoconcepto y autoestima: ¿sinónimos o constructos complementarios?”, en PSOCIAL. Revista de Investigación en Psicología Social, vol. 3 , núm. 1, Argentina: Universidad de Buenos Aires.

Ortega, Tamara (2014), "Criminalización y concentración de la pobreza urbana en barrios segregados: Síntomas de guetización en La Pintana, Santiago de Chile”, en EURE (Santiago), vol. 40, núm. 120, Chile: Pontificia Universidad Católica de Chile.

Pacheco, Berenice (2018), "Violencia escolar: la perspectiva de estudiantes y docentes", en Revista Electrónica de Investigación Educativa, vol. 20, núm. 1, México: Instituto de Investigación y Desarrollo Educativo.

Pereda, Noemí y Forns, María (2007), "Prevalencia y características del abuso sexual infantil en estudiantes universitarios españoles", en Child Abuse \& Neglect, vol. 31, Estados Unidos: Elsevier.

Pereda, Noemí et al. (2014), "Victimization and polyvictimization of Spanish children and youth: Results from a community sample”, en Child Abuse \& Neglect, vol. 38, núm. 4, Estados Unidos: Elsevier.

Pérez-Archundia, Eduardo y Gutiérrez-Méndez, David (2016), "El conflicto en las instituciones escolares”, en Ra Ximhai, vol. 12, núm. 3, México: Universidad Autónoma Indígena de México.

Primera Encuesta Nacional sobre Polivictimización en Niños, Niñas y Adolescentes (2017), Base EPV 2017, Chile: Centro de Estudios y Análisis del Delito.

Pinto-Cortez, Cristian et al. (2017), "Estudios sobre violencia interpersonal contra la infancia y la adolescencia en Chile: una revisión”, en Interciencia, vol. 42, núm. 5, Venezuela: Asociación Interciencia.

Pitch, Tamar (2009), La sociedad de la prevención, Argentina: Ad-Hoc.

Radford, Lorrain et al. (2013), "The prevalence and impact of child maltreatment and other types of victimization in the UK: Findings from a population survey of caregivers, children and young people and young adults", en Child Abuse \& Neglect, vol. 37, núm. 10, Estados Unidos: Elsevier.

Rodríguez, Carlos et al. (2017), "Sobre el miedo al delito y los otros miedos: el ciudadanovíctima y la inseguridad transversalizada”, en Politica y Sociedad, vol. 54, núm. 3, España: Universidad Complutense de Madrid.

Salgado, Cecilia (2012), "Revisión de las investigaciones acerca del bullying: desafíos para su estudio”, en Benites, Luis et al., Bullying y convivencia en la escuela. Aspectos conceptuales, aplicativos y de investigación, Perú: Dennis Morzán.

Salmerón, Jordi (2011), "Superación del modelo anterior de justicia juvenil (tutelar) por el actual modelo (de responsabilidad). ¿Se lo ha creído alguien?”, en Revista Critica Penaly Poder, núm. 1. Disponible en: https://revistes.ub.edu/index.php/CriticaPenalPoder/ article/viewFile/1991/2087 [29 de agosto de 2020]. 
Samper-García, Paula (2015), "Victimización en la escuela: relación de la crianza y variables funcionales-disfuncionales del desarrollo", en Anales de Psicología, vol. 31, núm. 3, doi: 10.6018/analesps.31.3.173291. Disponible en: https://revistas.um.es/analesps/ article/view/analesps.31.3.173291 [26 de agosto de 2020].

Silva, Daniel et al. (2017), "Intento de suicidio y factores de riesgo en una muestra de adolescentes escolarizados de Chile", en Revista de Psicopatología y Psicología Clínica, vol. 22, núm. 1, España: Asociación Española de Psicología Clínica y Psicopatología.

Tijmes, Cecilia (2012), "Violencia y clima escolar en establecimientos educacionales en contextos de alta vulnerabilidad social de Santiago de Chile", en Psybke, vol. 21, núm. 2, Chile: Pontificia Universidad Católica de Chile.

Tocornal, Ximena et al. (2014), "Delincuencia y violencia en entornos residenciales de Santiago de Chile", en Revista de Geografía Norte Grande, vol. 57, Chile: Pontificia Universidad Católica de Chile.

Tovar, Aída et al. (2016), "El maltrato infantil desde la voz de la niñez", en Revista Mexicana de Ciencias Agrícolas, vol. 7, núm. 1, México: Instituto Nacional de Investigaciones Forestales, Agrícolas y Pecuarias.

Trucco, Daniela e Inostroza, Pamela (2017), Las violencias en el espacio escolar, Chile: Naciones Unidas.

Valdenegro, Boris y Calderón-Flández, Claudia (2016), "Alteridad, infancia y delincuencia: análisis discursivo desde programas sociales hacia infancias en Chile”, en Revista Latinoamericana de Ciencias Sociales, Niñez y Juventud, vol. 14, núm. 2, Colombia: Universidad de Manizales.

Valdivieso, Carlos (1999), "Violencia y delincuencia en Chile", en Perspectivas en Politica, Economia y Gestión, vol. 3, núm. 1, Chile: Universidad de Chile. 
Anexo

Tabla 1

Descriptivos (media) y porcentajes de ocurrencia por dimensión del Cuestionario de Victimización Juvenil según tiempo del suceso

\begin{tabular}{|c|c|c|c|}
\hline & Durante la vida & \multicolumn{2}{|c|}{ Último año } \\
\hline Dimensiones & $\%$ & $\%$ & Fx \\
\hline Delitos comunes $[\%(\mathrm{x})]$ & $77,0(, 373)$ & 51,0 & $(, 278)$ \\
\hline - Robo sin uso de fuerza & - & 20,4 & 46,0 \\
\hline - Destrozo de objetos personales & - & 18,3 & 57,9 \\
\hline - Ataque físico sin uso de armas & - & 28,5 & 68,1 \\
\hline Efectuadas por cuidadores $[\%(\mathrm{x})]$ & $51,8(, 399)$ & \multicolumn{2}{|c|}{$34,8(, 354)$} \\
\hline - Insultos, dichos crueles o desapego & - & 28,4 & 82,2 \\
\hline - Daño físico por algún adulto cercano & - & 12,8 & 63,4 \\
\hline Efectuadas por pares $[\%(\mathrm{x})]$ & $61,5(, 354)$ & \multicolumn{2}{|c|}{$28,4(, 291)$} \\
\hline $\begin{array}{l}\text { - Ataques físicos por otro NNA } \\
\text { - Insultos, molestia o aislamiento por parte de } \\
\text { otros NNA }\end{array}$ & - & $\begin{array}{l}14,8 \\
14,1\end{array}$ & $\begin{array}{l}53,1 \\
80,5\end{array}$ \\
\hline Sexuales $[\%(\mathrm{x})]$ & $26,4(, 226)$ & \multicolumn{2}{|c|}{$16,7(, 186)$} \\
\hline $\begin{array}{l}\text { - Prácticas sexuales consentidas con mayores } \\
\text { de edad } \\
\text { - Dichos sexuales sobre la persona o el cuerpo }\end{array}$ & - & $\begin{array}{l}7,9 \\
6,1\end{array}$ & $\begin{array}{l}77,1 \\
66,4\end{array}$ \\
\hline Indirectas $[\%(\mathrm{x})]$ & $83,3(, 409)$ & \multicolumn{2}{|c|}{$32,2(, 311)$} \\
\hline $\begin{array}{l}\text { - Testigo de balaceras, microtráfico y/o peleas } \\
\text { de pandillas } \\
\text { - Presenciar una discriminación } \\
\text { - Testigo de ataque físico intencionado sin uso } \\
\text { de armas }\end{array}$ & $\begin{array}{l}- \\
-\end{array}$ & $\begin{array}{l}45,3 \\
42,9 \\
31,7\end{array}$ & $\begin{array}{l}87,1 \\
87,7 \\
66,0\end{array}$ \\
\hline Digitales $^{2}[\%]$ & 32,3 & \multicolumn{2}{|c|}{20,9} \\
\hline $\begin{array}{l}\text { - Acoso o rumores malintencionados en Internet } \\
\text { - Preguntas o chat sexuales que hacen sentir } \\
\text { incómodo }\end{array}$ & $\begin{array}{l}24,1 \\
17,7\end{array}$ & $\begin{array}{l}14,1 \\
11,8\end{array}$ & $\begin{array}{l}77,7 \\
70,3\end{array}$ \\
\hline
\end{tabular}

Notas: ${ }^{2} \mathrm{La}$ dimensión sólo cuenta con dos indicadores, por lo que solamente se consigna su frecuencia relativa; $\mathrm{Fx}=$ ocurrencia del hecho más de dos veces en el último año, representada en porcentaje; $(\mathrm{x})=$ media estandarizada de los indicadores que componen la dimensión; se representan los ítems de mayor recurrencia. Fuente: Elaboración propia con datos de la Primera Encuesta Nacional de Polivictimización (2017). 


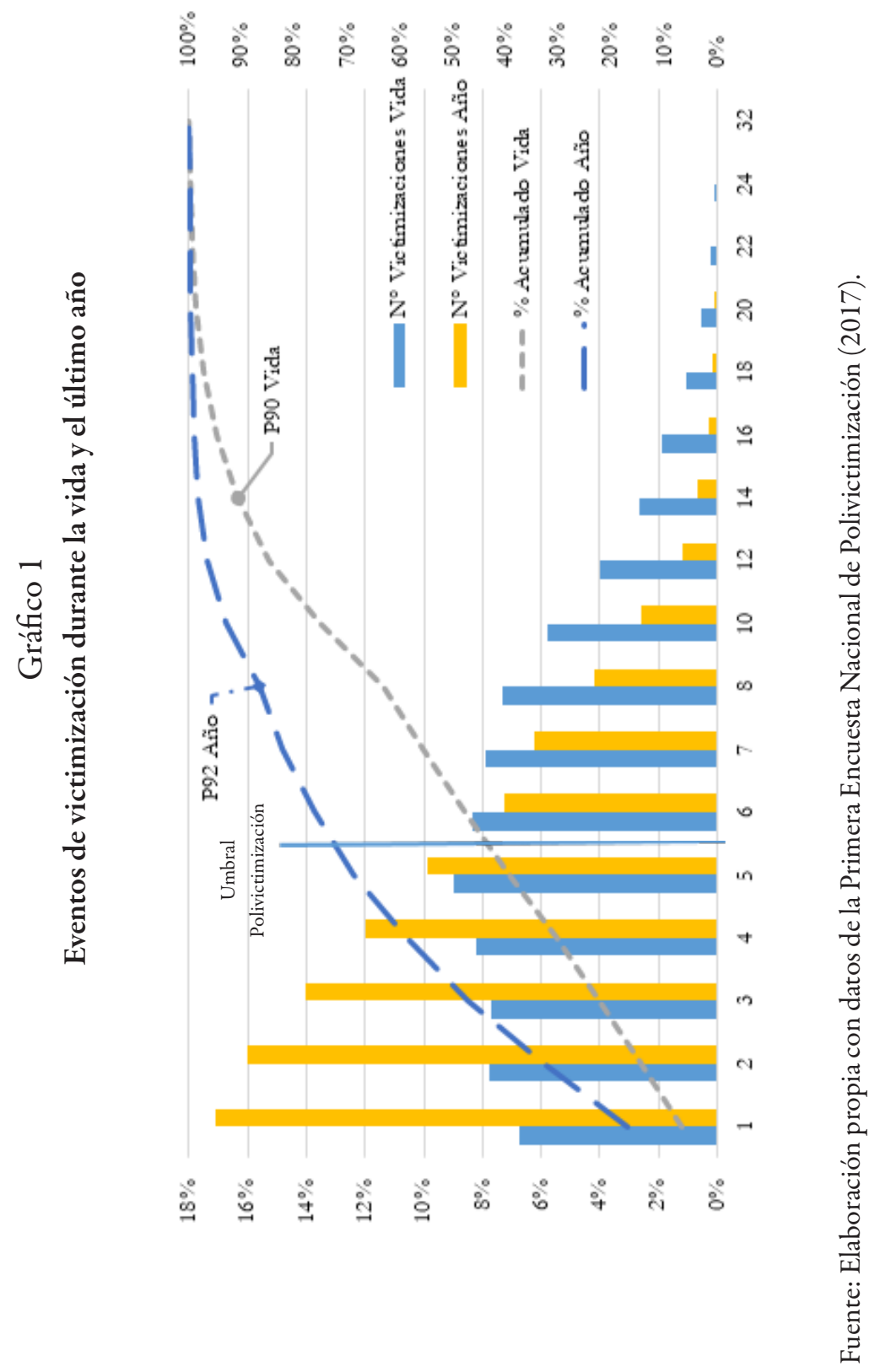




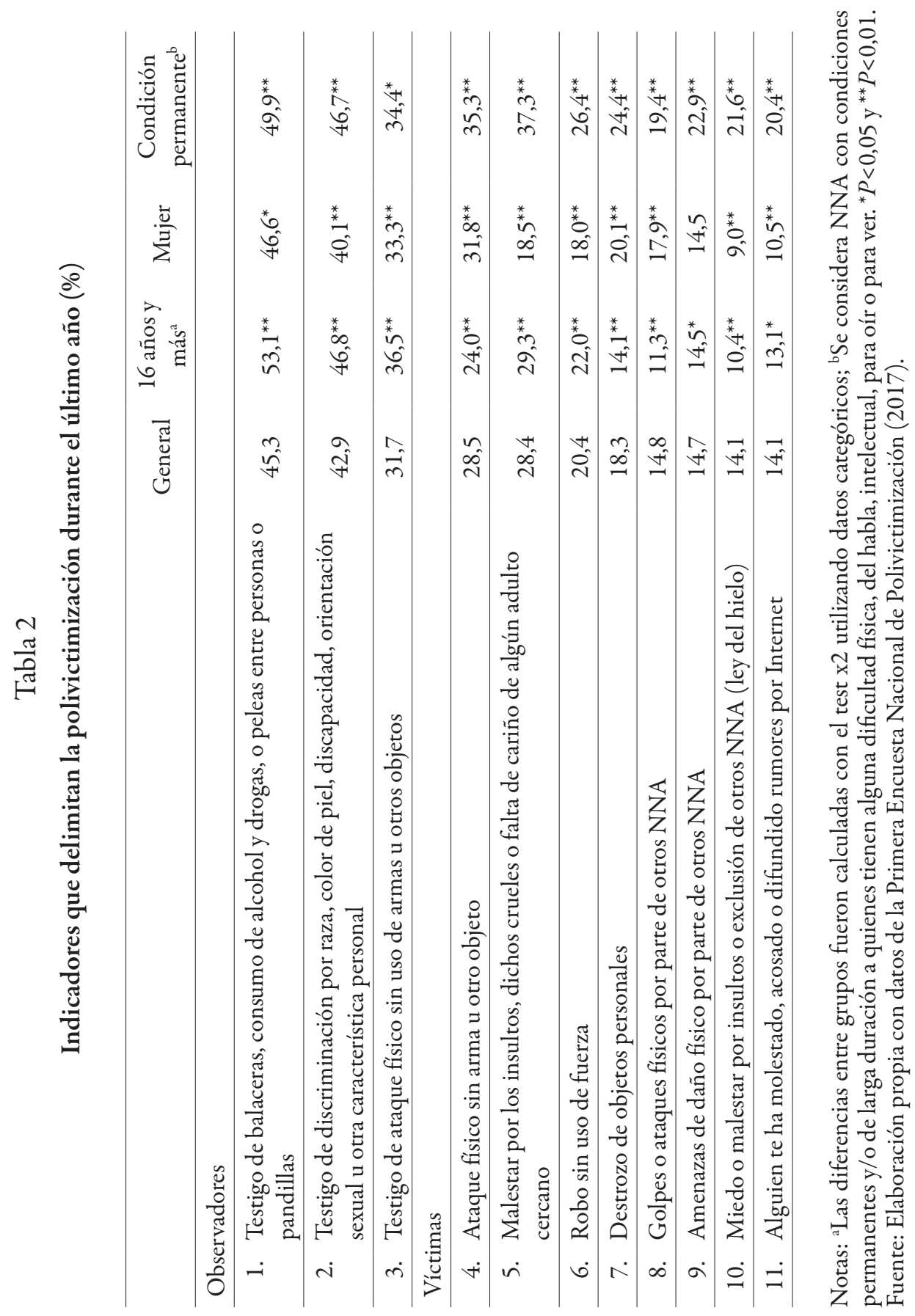




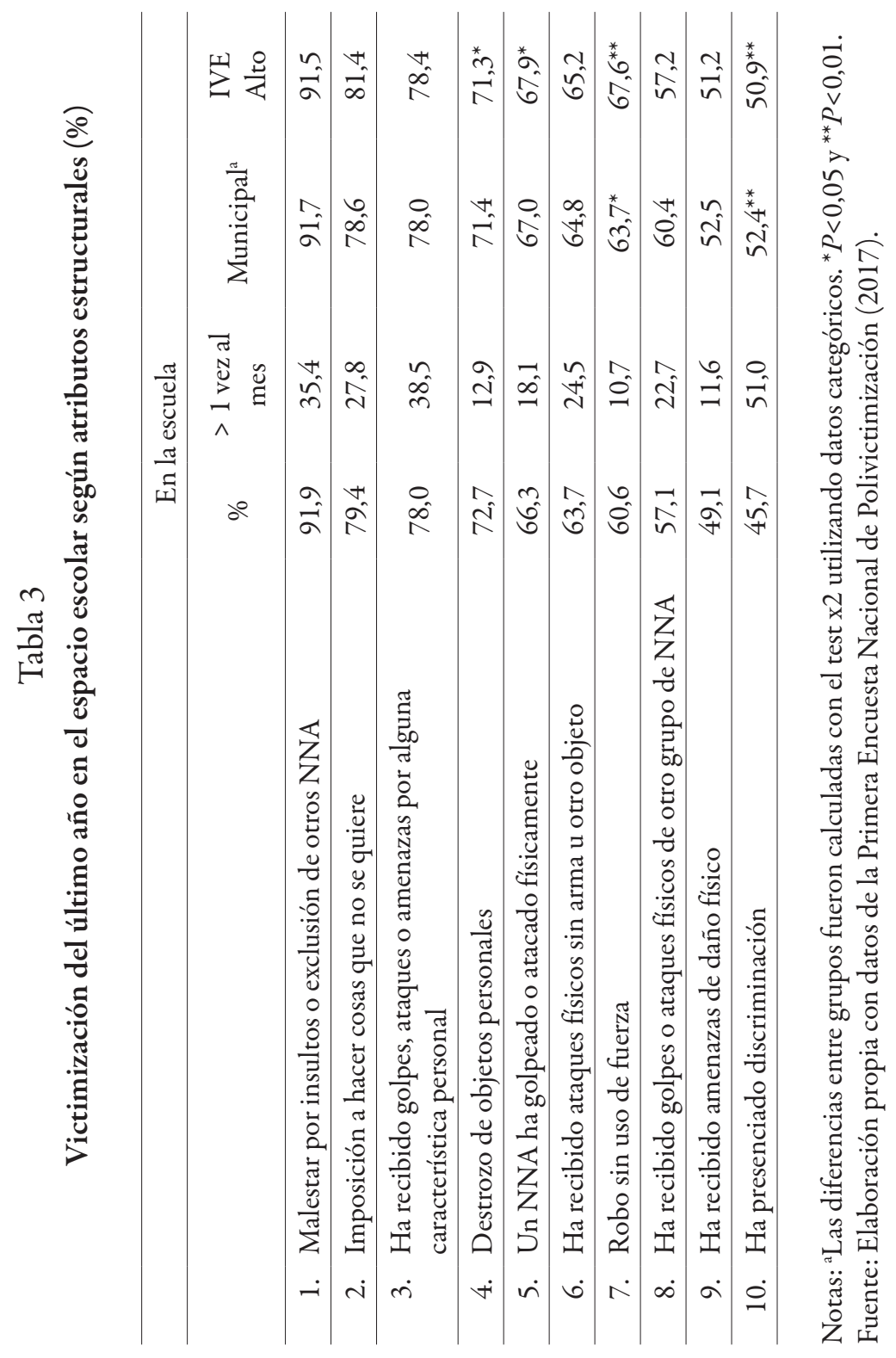


Carlos Rodríguez Garcés. Doctor por la Universidad de Barcelona, docente en la Universidad del Bío-Bío, director del Centro de Investigación Educativa (CIDCIE), Chillán, Chile. Líneas de investigación: educación, tecnologías de la información y familia. Publicaciones recientes: Rodríguez Garcés, Carlos y Padilla Fuentes, Geraldo (2020), “Cáncer mamario y cervicouterino: el problema femenino en la acción preventiva”, en Revista Universidad y Salud, vol. 22, núm. 1, Colombia: Universidad de Nariño. Rodríguez Garcés, Carlos y Padilla Fuentes, Geraldo (2020), “¡Y cómo nos va en matemáticas?: La calidad de la influencia de pares y la predisposición personal hacia el aprendizaje en un contexto de segmentación socioeducativa”, en Revista Educación Matemática, vol. 32, núm. 1, México: Sociedad Mexicana de Investigación y Divulgación de la Educación Matemática. Rodríguez Garcés, Carlos y Padilla Fuentes Geraldo (2020), "Cuidarlos en casa o pre escolarizarlos: itinerarios de una decisión materna por medio de árboles de segmentación”, en Revista Universidad y Sociedad, vol. 11, núm. 4, Cuba: Universidad de Cienfuegos.

Geraldo Padilla Fuentes. Trabajador social, licenciado en Trabajo Social, investigador adscrito al Centro de Investigación Educativa (CIDCIE), Universidad del Bío-Bío, Chillán, Chile. Líneas de investigación: educación y sociedad. Publicaciones recientes: Rodríguez Garcés, Carlos y Padilla Fuentes, Geraldo (2020), "Cáncer mamario y cervicouterino: el problema femenino en la acción preventiva”, en Revista Universidad y Salud, vol. 22, núm. 1, Colombia: Universidad de Nariño. Rodríguez Garcés, Carlos y Padilla Fuentes, Geraldo (2020), “¿Y cómo nos va en matemáticas?: La calidad de la influencia de pares y la predisposición personal hacia el aprendizaje en un contexto de segmentación socioeducativa”, en Revista Educación Matemática, vol. 32, núm. 1, México: Sociedad Mexicana de Investigación y Divulgación de la Educación Matemática. Rodríguez Garcés, Carlos y Padilla Fuentes Geraldo (2020), "Cuidarlos en casa o pre escolarizarlos: itinerarios de una decisión materna por medio de árboles de segmentación", en Revista Universidad y Sociedad, vol. 11, núm. 4, Cuba: Universidad de Cienfuegos. 
Denisse Espinosa Valenzuela. Profesora de Educación Media en Castellano y Comunicación. Investigadora Adscrita al Centro de Investigación Educativa (CIDCIE), Universidad del Bío-Bío, Chillán, Chile. Líneas de investigación: educación y sociedad. Publicaciones recientes: Rodríguez Garcés, Carlos Padilla, Fuentes, Geraldo y Espinosa Valenzuela, Denisse (2020), "Sentido de pertenencia escolar entre niños, niñas y adolescentes en Chile: perfiles e itinerarios mediante árboles de clasificación", en Revista Colombiana de Educación, núm. 81, Colombia: Universidad Pedagógica Nacional. Rodríguez Garcés, Carlos et al. (2021), "No todo es Prueba de Selección Universitaria: el ranking como vía de inclusión a la universidad en Chile", en Sophia, vol. 17, núm. 2, Colombia: Universidad la Gran Colombia. Rodríguez Garcés, Carlos et al. (2020), "Dónde quiero que estudien mis hijos/as: caracterización de la oferta educativa y sus niveles de demanda en Chile", en REXE. Revista de Estudios y Experiencias en Educación, vol. 19, núm. 41, Chile: Facultad de Educación de la Universidad Católica de la Santísima Concepción. 